\title{
Quantifying Damage Accumulation Using State-of-The-Art FFT Method
}

\author{
R. Pokharel ${ }^{a}$, S. F. Li*, J. Lind ${ }^{*}$, C. M. Hefferan*, U. Lienert ${ }^{\dagger}$, \\ R. A. Lebensohn ${ }^{+}$, R.M. Suter*, A. D. Rollett \\ Materials Sci. \& Eng., *Physics Dept., Carnegie Mellon Univ., PA-15213, USA; \\ ${ }^{\dagger}$ Argonne National Laboratory, 9700 S. Cass Avenue, Lemont, IL 60439, USA; \\ ${ }^{+}$Los Alamos National Laboratory, NM 87545, USA. \\ ${ }^{\mathrm{a}}$ reeju@cmu.edu
}

Keywords: FFT simulation, viscoplastic deformation, 3D microstructure, HEDM

\begin{abstract}
A 3D microstructure, measured by high-energy x-ray diffraction microscopy, is used as an input to a parallelized viscoplastic Fast Fourier Transform code (VPFFT) to simulate a tensile test. Distributions of strain, damage accumulation, neighbor interactions, and Schmid factor mismatch throughout the microstructure are calculated. These results will form the basis of a direct comparison to microstructure maps that track plastic deformation in the real sample.
\end{abstract}

\section{Introduction}

Microstructure plays an important role in determining the properties of polycrystalline materials. Our main goal here is to understand both the microstructural and mechanical aspects of initiation of ductile failure in materials. Synchrotron radiation based High Energy Diffraction Microscopy (HEDM) [1,2], combined with tomography, has made it possible to non-destructively probe bulk materials and obtain both spatial as well as orientation information, which are invaluable in understanding the relationship between microstructure and damage accumulation during the early stages of failure. We use such a measurement of a $\mathrm{Cu}$ wire as the starting point for computational modeling, using recently developed and parallelized viscoplastic Fast Fourier Transform (VPFFT) code to simulate a tensile test. These calculations will be directly compared to HEDM maps of the deformed microstructures once they become available.

\section{Experimental}

In-situ measurements were performed at beam line 1-ID at the Advanced Photon Source at Argonne National Laboratory. A $1 \mathrm{~mm}$ diameter, $99.9999 \%$ pure $\mathrm{Cu}$ wire was selectively electropolished in the middle of the length to neck it down to $210 \mu \mathrm{m}$ diameter. A $300 \mu \mathrm{m}$ long section of the wire was mapped to yield a three dimensional crystallographic orientation field.

\section{Simulation}

Simulation of viscoplastic deformation using the VPFFT method was conducted in order to compare experimental and simulated results. The VPFFT method has been described extensively elsewhere $[3,7,8]$. Experimental data was fed in as an input to the simulation and uniaxial deformation was modeled up to $50 \%$ strain with $1 \%$ strain steps. From the analysis, the relationship between stress gradient, average misorientation, distance from grain boundaries, and Schmid factor mismatch were quantified.

\section{Results and Discussions}

Using the measured microstructure from the HEDM experiment as an input to VPFFT, local fluctuations in stress and strain were simulated up to $50 \%$ strain. Fig. 1 shows the orientation, misorientation, and stress maps obtained from VPFFT simulation for 3\%, 10\%, and 17\% strain steps. The 2D sections were extracted from the necked region of each simulated microstructure. This allowed us to make a qualitative analysis on the change in internal structures, damage accumulations, and stress concentrations as the strain increased in the material. 


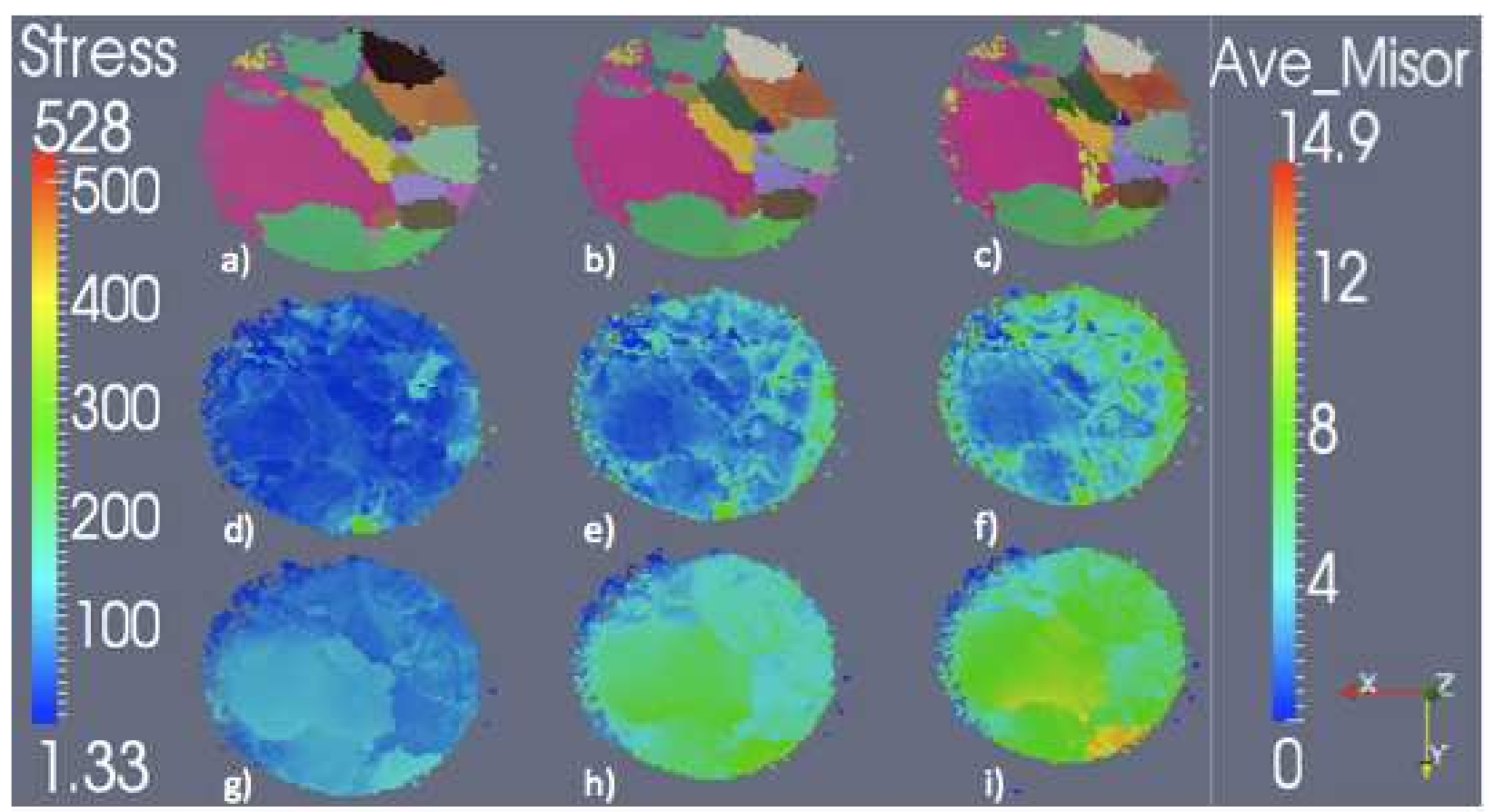

Fig.1: Orientation, misorientation and stress maps for 3\%, 10\% and 17\% strains (left to right). (a)(c) Orientation maps. (d)-(f) Misorientation maps. (g)-(i) Stress field (units in MPa) maps.

In Fig.1.(a)-(c), each color represents a different orientation in Rodriguez-Frank (RF) space. The maps show that new orientations gradually develop as we increase the strain, which is evident at $17 \%$ strain. Fig 1.(d)-(f) show the intra-granular misorientation development as we go higher in strain, which can also be associated with the deformation field developed during uniaxial tensile test [4]. Intra-granular misorientations arise due to the dislocation pile up within each grain in the microstructure. Fig.1 (g)-(i) show the von-misses equivalent stress fields, for different deformation states. From these maps, it appears that stress and misorientation distributions show systematic variation throughout the microstructure. The results obtained from VPFFT simulation will be validated against the experimental data, once available.

From the literature, we know that misorientation and stress field are correlated [4]. We are interested in understanding the initiation of failure, which can be hypothesized to be occurring at regions of high stresses and large misorientations. Fig. 2 plots the distance from the grain boundary versus kernel average misorientation (KAM) and stress gradient. These plots were generated for simulated results at the $50 \%$ strain step. Higher misorientation develops near grain boundaries, and decreases rapidly as we move farther away from the boundary. A similar correlation was observed between the stress gradient, which is calculated as the root mean square average of the difference in five stress components, and relative distance from the boundary. 

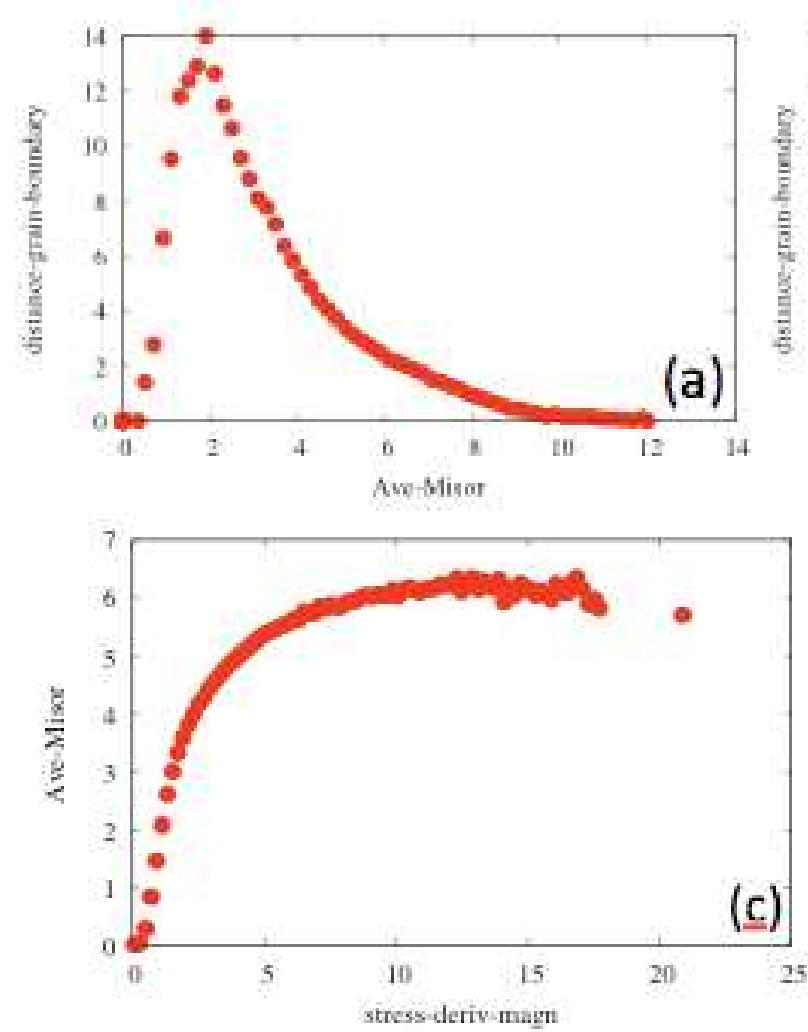

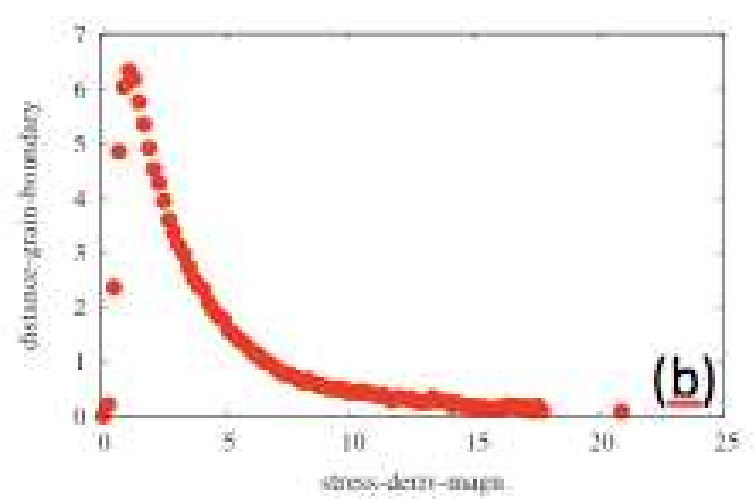

Fig. 2: Correlation plots. a) $\&$ b) Distance from grain boundary versus average misorientation and stress gradient, respectively. c) Average misorientation versus magnitude of stress gradient.

Furthermore, Fig. 2c shows the relation between the average misorientation and gradient in stress, which is in agreement with the literature [4]. Thus, misorientation can also be seen as a measure of stress accumulation in the microstructure. These plots indicate that high deformation fields develop around grain boundaries, which are most likely to serve as failure initiation sites.
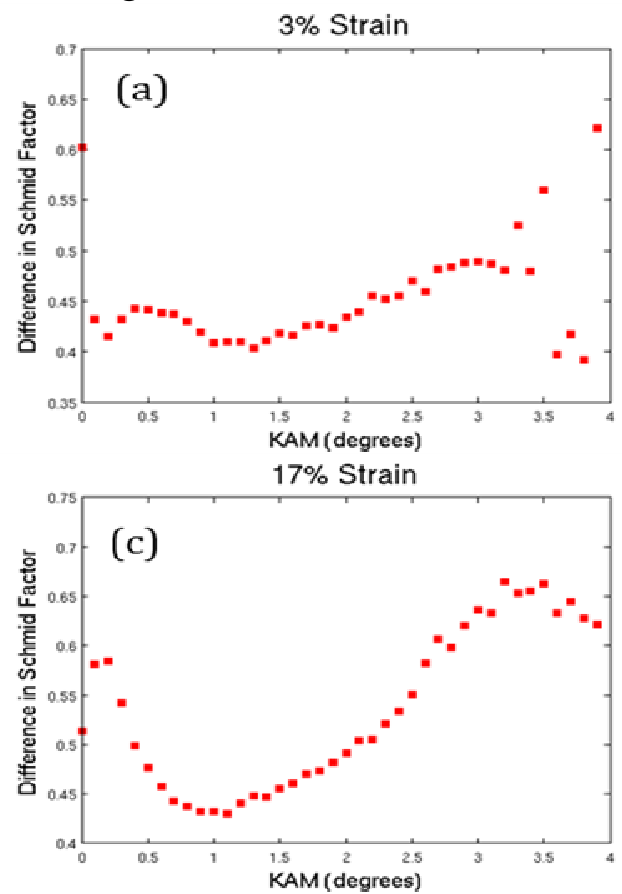

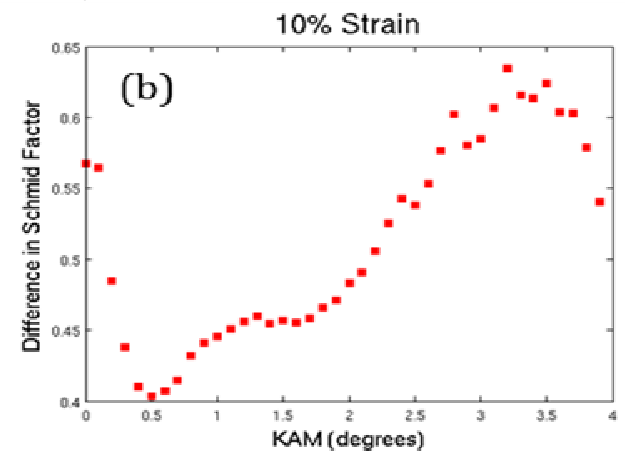

Fig. 3: Difference in Schmid factor versus kernel average misorientation. (a) $3 \%$ strain. (b) $10 \%$ strain.

In polycrystalline materials, grain interactions are important in understanding the fundamentals of failure initiation mechanisms, in the microstructure [5,6]. For one of our initial failure analysis attempts, a simple single crystal model is applied to study the orientation dependence on slip activation, and its relationship to the surrounding environment. Schmid factor mismatches were calculated between neighboring grains from VPFFT data. Additionally, only points adjacent to the grain boundaries were included in the KAM calculation. In Fig. 3, we have plotted the difference in Schmid factor between neighboring grains versus the KAM for different strain steps. For a coarsely 
binned data, there appears to develop some linear relationships with strain, but overall no simple correlations were observed; thus, detailed statistical analysis might be required to further predict any significant relationship if present.

\section{Summary}

HEDM [1, 2] was used to provide an initial microstructure as input to a VPFFT simulation with the objective of simulating deformation and, eventually, the failure initiation process in ductile materials $[3,7]$. High stresses and misorientations were observed near grain boundaries at small strains, but these correlations weaken as the strain level increases. No significant correlation was found between the Schmid factor mismatch against KAM across different strain states. However, a strong correlation was seen between the stress gradient and the average misorientation with the distance from grain boundaries, which are possible failure initiation sites. These preliminary results point to the efficacy of combining experiment with simulations with the aim of more effectively and accurately predicting failure in relation to microstructure.

\section{Acknowledgement}

This work was supported by DOE Award number DE-FG02-09ER46645. Use of the Advanced Photon Source was supported by the U. S. Department of Energy, Office of Science, and Office of Basic Energy Sciences, under Contract No. DE-AC02-06CH11357.

\section{References:}

[1] R. M. Suter, C. Hefferan, S. F. Li, D. Hennessy, C. Xiao, U. Lienert, and B. Tieman, "Probing Microstructure Dynamics With X-Ray Diffraction Microscopy", J. Eng. Materials Tech. 130 (2008) 021007.

[2] C. M. Hefferan, S. F. Li, J. Lind, U. Lienert, A. D. Rollett, P. Wynblatt and R. M. Suter (2009). "Statistics of High Purity Nickel Microstructure From High Energy X-ray Diffraction Microscopy." CMC - Computers Materials \& Continua 14 (2009) 209-219.

[3] R. A. Lebensohn, R. Brenner, O. Castelnau and A. D. Rollett, "Orientation image-based micromechanical modelling of subgrain texture evolution in polycrystalline copper." Acta Materialia 56 (2008) 3914-3926.

[4] A.D. Rollett, R.A. Lebensohn, M. Groeber, Y. Choi, J. Li and G.S. Rohrer, "Stress Hot Spots in Viscoplastic Deformation of Polycrystals.” Model. Simul. Mater. Sci. Eng. 18 (2010) 074005.

[5] U. Lienert, T.-S. Han, J. Almer, P.R. Dawson, T. Leffers, L. Margulles, S. F. Nielsen, H.F. Poulsen, and S. Schmidt, "Investigating the effect of grain interaction during plastic deformation of copper", Acta Materialia 52 (2004): 4461-4467.

[6] S. K. Mishra, P. Pant, K. Narasimhan, A. D. Rollett, and I. Samajdar "On the widths of orientation gradient zones adjacent to grain boundaries", Scripta Materialia 61 (2009) 273-276.

[7] R. A. Lebensohn, Y. Liu, P. Ponte Castaneda, "On the accuracy of the self-consistent approximation for polycrystals: comparison with full-filed numerical simulations", Acta Materialia 52 (2004) 5347-5361.

[8] R. A. Lebensohn (2001), "N-Site Modeling of a 3D Viscoplastic Polycrystal Using Fast Fourier Transform”, Acta Materialia 49 (2001) 2723-2737. 


\section{Textures of Materials}

10.4028/www.scientific.net/MSF.702-703

Quantifying Damage Accumulation Using State-of-the-Art FFT Method 10.4028/www.scientific.net/MSF.702-703.515 